\title{
Survival of Patients With Cervical Cancer in Rural India
}

\author{
Jissa Vinoda Thulaseedharan a, b, Nea Malila ${ }^{\mathrm{b}, \mathrm{c}}$, Rajaraman Swaminathan ${ }^{\mathrm{d}}$, Pulikottil Okuru Esmy ${ }^{\mathrm{e}}$, \\ Matti Hakama ${ }^{\text {b, c }}$, Richard Muwonge ${ }^{f, g}$, Rengaswamy Sankaranarayanan ${ }^{f}$
}

\begin{abstract}
Background: Patients' survival after diagnosis of cervical cancer is indirectly influenced by socio-economic factors. We evaluated this survival and its socio-economic determinants in a rural population in south India.
\end{abstract}

Methods: We assessed 165 women diagnosed with cervical cancer from the routine care control arm of a randomized screening trial conducted in rural south India. Kaplan-Meier curves were plotted to illustrate the observed survival of cancer patients. The effect of socio-economic factors was assessed using Cox proportional hazards regression analysis.

Results: The 5-year observed survival was 32.5\%, ranging from $9 \%$ for stage IV to $78 \%$ for stage I cancers. Women with poor socioeconomic status (SES) had up to a $70 \%$ higher risk of death. Higher household income was significantly associated with poorer survival. However, most women in the higher income group were married women and housewives, hence with no personal income.

Conclusion: Cervical cancer survival was disappointingly low in these rural populations of India and stage of disease at diagnosis was the strongest determinant. A higher household income is not always associated with women being empowered in terms of seeking healthcare. The study findings further stress the importance of strengthening prevention and screening opportunities to women in rural popula-

Manuscript accepted for publication October 21, 2015

${ }^{a}$ Achutha Menon Centre for Health Science Studies (AMCHSS), Sree Chitra Tirunal Institute for Medical Sciences and Technology (SCTIMST), Trivandrum, India

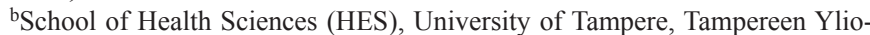
pisto, Finland

${ }^{c}$ Finnish Cancer Registry, Pieni Roobertinkatu, Helsinki, Finland

${ }^{\mathrm{d} B i o s t a t i s t i c s}$ and Cancer Registry, Cancer Institute (WIA), Chennai, India

${ }^{\mathrm{e}}$ Christian Fellowship Community Health Centre (CFCHC), Ambillikai, Tamil

Nadu, India

fScreening Group, International Agency for Research on Cancer (IARC), Lyon, France

'Corresponding Author: Richard Muwonge, Screening Group, International Agency for Research on Cancer, 150 Cours Albert Thomas, 69372 Lyon Cedex 08, France. Email: MuwongeR@iarc.fr

doi: http://dx.doi.org/10.14740/jcgo367w tions.

Keywords: Cervical cancer; Survival; Rural India; Socio-economic determinants

\section{Introduction}

Incidence and mortality estimates are used to measure the burden of cancer in a population and survival estimates are ideal for evaluating the outcome of cancer control activities [1]. Survival studies evaluate the quality and quantity of life of a group of patients after diagnosing the disease [2]. Longterm survival, usually for more than 5 years, reflects cure and is considered a positive measure to evaluate the efficiency of the health system [3].

Globally, cervical cancer survival varies widely between countries. The 5-year survival rates for women diagnosed with cervical cancer during 1995 - 1999 in developed countries varied from $50 \%$ to $70 \%$ [4]. For Africa, Asia, and Central America, the lowest survival rates during 1990 - 2001 were observed in Uganda and Gambia with a 5-year age standardized relative survival (ASRS) of 19\% and 23\%, respectively, and the highest survival rates were reported in China, Singapore, South Korea, and Turkey, where the ASRS varied from $63 \%$ to $79 \%$ [5]. The median 5-year ASRS for cervical cancer in India was $46 \%$ ranging from $34 \%$ to $60 \%$ [5].

The remarkable inequality in cancer survival between and within countries is largely due to the differences in general awareness, availability of early detection practices, trained human resources, and accessibility to cancer services, diagnosis and treatment [3]. Age at diagnosis, aggressiveness and clinical extent of cancer, willingness and determination of the patient to complete his/her treatment and socio-economic factors also influence survival from cancer [1]. Even though cervical cancer is considered an eminently preventable cancer, late stage diagnosis and delay in getting treatment lead to reduced cervical cancer survival in low resources settings [6]. Thus, the inequality in cervical cancer survival is related to the differences in medical, biological, cultural, genetic, geographic, and socio-economic factors [7-9].

To assess the socio-economic factors that affect cervical cancer survival in a rural population of south India, we used 
Table 1. Characteristics and Survival Experiences of Women Diagnosed With Cervical Cancer From the Control Arm During 2000 - 2006 and Followed Until December 31, 2011

\begin{tabular}{|c|c|c|c|c|c|}
\hline \multirow{2}{*}{ Women's characteristics } & \multirow{2}{*}{$\begin{array}{l}\text { Number of women } \\
\text { with cancer n }(\%)\end{array}$} & \multicolumn{3}{|c|}{ Observed survival (\%) at } & \multirow{2}{*}{ P-value } \\
\hline & & 1-year & 3-year & 5-year & \\
\hline Total & 165 & 67.3 & 40 & 32.5 & \\
\hline \multicolumn{6}{|l|}{ Individual } \\
\hline \multicolumn{6}{|l|}{ Age at diagnosis } \\
\hline $30-39$ & $37(22.4)$ & 70.3 & 45.9 & 37.3 & \\
\hline $40-49$ & $53(32.1)$ & 66.0 & 39.6 & 34.0 & \\
\hline $50+$ & $75(45.5)$ & 66.7 & 37.3 & 29.1 & 0.5458 \\
\hline \multicolumn{6}{|l|}{ Stage of disease } \\
\hline Stage I & $18(10.9)$ & 88.9 & 83.3 & 77.6 & \\
\hline Stage II & $32(19.4)$ & 68.8 & 37.5 & 21.4 & \\
\hline Stage III & $62(37.6)$ & 69.4 & 35.4 & 25.3 & \\
\hline Stage IV & $11(6.7)$ & 36.4 & 9.1 & 9.1 & \\
\hline Unknown & $42(25.5)$ & 61.9 & 38.1 & 35.7 & 0.0023 \\
\hline \multicolumn{6}{|l|}{ Education* } \\
\hline No schooling & $145(87.9)$ & 66.9 & 37.9 & 30.9 & \\
\hline Some schooling & $17(10.3)$ & 82.4 & 64.5 & 51.6 & 0.1794 \\
\hline \multicolumn{6}{|l|}{ Occupation } \\
\hline House wife/others & $60(36.4)$ & 73.3 & 48.3 & 36.4 & \\
\hline Manual & $105(63.6)$ & 63.8 & 35.2 & 30.3 & 0.3117 \\
\hline \multicolumn{6}{|l|}{ Marital status } \\
\hline Currently married & $146(88.5)$ & 66.4 & 39.7 & 31.3 & \\
\hline Widowed/separated & $19(11.5)$ & 73.7 & 42.1 & 42.1 & 0.4668 \\
\hline \multicolumn{6}{|l|}{ Household } \\
\hline \multicolumn{6}{|l|}{ Type of house } \\
\hline Thatched & $27(16.4)$ & 48.2 & 29.6 & 25.9 & \\
\hline Tiled/concrete & $138(83.6)$ & 71.0 & 42.0 & 33.8 & 0.2588 \\
\hline \multicolumn{6}{|l|}{ Income (INR) } \\
\hline$<2,000$ & $117(70.9)$ & 69.2 & 43.5 & 38.2 & \\
\hline $2,000+$ & $48(29.1)$ & 62.5 & 31.3 & 18.8 & 0.0143 \\
\hline
\end{tabular}

*The information on education is missing for three observations. INR: Indian Rupees. 50 INR was equivalent to 1 US dollar during $2000-2003$.

the material consisting of 165 women diagnosed with cervical cancer during 2000 - 2006 from a cohort of 31,000 women in the control arm of a large VIA screening trial.

\section{Materials and Methods}

The details of the base population and the screening trial were described in earlier papers [10-12]. This trial aimed to evaluate the effect of a single round of VIA screening on cervical cancer incidence and mortality. The trial protocol was reviewed and approved by the institutional scientific and ethical committees of Christian Fellowship Community Health Centre (CFCHC) and the International Agency for Research on Cancer (IARC).
Clusters of eligible women aged 30 - 59 years, with an intact uterus and no history of cancer, were randomized into intervention and control arms during 2000 - 2003. Before enrolment, the trial was explained to the participants in the local language and a signed informed consent was obtained. A structured questionnaire was used to obtain the participants' demographic and socio-economic characteristics. Women in the intervention arm were offered screening with VIA, whereas those in the control arm were informed about screening, symptoms and risk factors of cervical cancer, where the screening, early diagnosis and treatment facilities are available, and advised to utilize such routine healthcare facilities.

The trial population was followed annually until December 2006 to collect information on death, migration and cervi- 


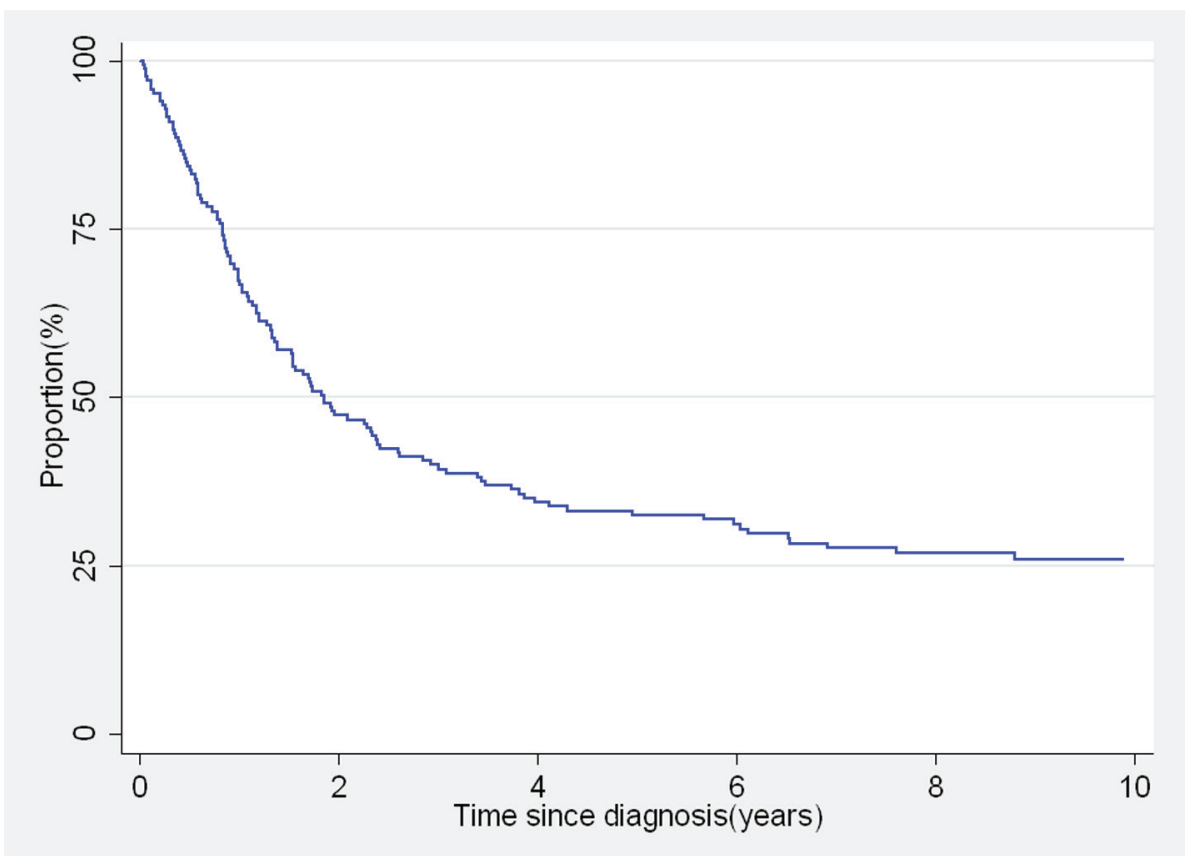

Figure 1. Kaplan-Meier overall survival curve of 165 women diagnosed with cervix cancer from the control arm.

cal cancers. The Dindigul Ambillikai Cancer Registry (DACR) staff actively collected information on women diagnosed with cervical cancer from the entire Dindigul district [13] and subsequently matched the information with the study database on a case-by-case basis to obtain all the incident cervical cancer cases diagnosed during 2000 - 2006 from the study population. In the final analysis, therefore, we used the updated information of 165 women diagnosed with cervical cancer during 2000 - 2006 from the control arm. The trial base population was again followed in 2011 - 2012 to collect information on death and migration.

The women's characteristics studied were age at diagnosis (categorized in $30-39,40-49$ and 50+), stage of disease (stage I, II, III, IV and unknown) and the baseline information on socio-economic factors such as formal education (no schooling and some schooling), occupation (housewife/other and manual workers), marital status (currently married and widowed/separated), type of house (thatched and tiled/concrete), and household income (categorized in $<2,000$ and 2,000+ Indian Rupees (INR)).

Data analysis was done using the Stata/IC 11.2 software package (Stata Corp LP, TX, USA). The primary endpoint was death from cervical cancer. To give each of the included patients a chance of having at least 5 years of survival, December 31, 2011 was used as the latest date of follow-up. For the definition of the study outcome, the vital status of all included patients by December 31, 2011 was established as dead, alive or lost to follow-up. Survival time was calculated from the date of diagnosis to date of death, for the patients who had died, or December 31, 2011 for those who were still alive or date of last seen for those lost to follow-up. Only two women were lost to follow-up. Kaplan-Meier curves were plotted to illustrate the observed survival of cancer patients and the log-rank test was used to test the equality of survivor functions. The effect of socio-economic factors was assessed using Cox proportional hazards regression analysis. Adjustment was made by including all the factors studied in a single regression model.

\section{Results}

Characteristics and survival experience of 165 women diagnosed with cervical cancer are presented in Table 1. Overall, $54.5 \%$ of the women were diagnosed with cervical cancer under the age of 50 years. Most women (63.6\%) were diagnosed in stage II or worse with a large number of women (37.6\%) in stage III and one-quarter of them had unknown stage at diagnosis. Only $10.3 \%$ of the women had had some education and $70.9 \%$ of women had low household income (i.e., $<2,000$ INR).

Kaplan-Meier survival curve for the overall survival of the 165 women with cervical cancer is shown in Figure 1. Over half of the patients did not survive for 2 years after diagnosis. The observed 1-, 3- and 5-year survival estimates were 67.3\%, $40 \%$ and $32.5 \%$, respectively (Table1). Survival estimates by age at diagnosis were $37.3 \%$ for $30-39$ years, $34 \%$ for 40 49 years and $29.1 \%$ for women aged 50 years and above $(\mathrm{P}=$ 0.5458 ). Women with formal education had a $20 \%$ higher difference in 5-year survival than women without formal education $(\mathrm{P}=0.1794$; Table 1). The survival estimates differences within the different categories of occupation, type of house and marital status also varied from $6 \%$ to $11 \%$ (Table 1 ).

There was a significant reduction in 5-year survival with the increasing stage at diagnosis; the survival estimates for stages I, II, III and IV were 77.6, 21.4, 25.3 and 9.1, respectively $(\mathrm{P}=0.0023$; Table 1$)$. These estimates resulted in the 
Table 2. Crude and Adjusted Hazard Ratios to Assess the Effect of Women Characteristics on Survival After Cervical Cancer DiagnosiS

\begin{tabular}{|c|c|c|}
\hline Women's characteristics & Crude hazard ratio $(95 \% \mathrm{CI})$ & Adjusted* hazard ratio $(95 \% \mathrm{CI})$ \\
\hline \multicolumn{3}{|l|}{ Individual } \\
\hline \multicolumn{3}{|l|}{ Age at diagnosis } \\
\hline $30-39$ & 1 & 1 \\
\hline $40-49$ & $1.13(0.68-1.90)$ & $1.24(0.73-2.14)$ \\
\hline $50+$ & $1.29(0.80-2.08)$ & $1.38(0.83-2.32)$ \\
\hline \multicolumn{3}{|l|}{ Stage of disease } \\
\hline Stage I & 1 & 1 \\
\hline Stage II & $3.15(1.36-7.31)$ & $3.81(1.54-9.40)$ \\
\hline Stage III & $3.12(1.40-6.91)$ & $3.67(1.56-8.64)$ \\
\hline Stage IV & $6.77(2.56-17.91$ & $6.74(2.34-19.02)$ \\
\hline Unknown & $3.00(1.31-6.83)$ & $3.51(1.44-8.54)$ \\
\hline \multicolumn{3}{|l|}{ Education } \\
\hline No schooling & 1 & 1 \\
\hline Some schooling & $0.64(0.33-1.23)$ & $0.71(0.35-1.40)$ \\
\hline \multicolumn{3}{|l|}{ Occupation } \\
\hline House wife/others & 1 & 1 \\
\hline Manual & $1.21(0.83-1.77)$ & $1.11(0.74-1.68)$ \\
\hline \multicolumn{3}{|l|}{ Marital status } \\
\hline Currently married & 1 & 1 \\
\hline Widowed/separated & $0.80(0.44-1.45)$ & $0.68(0.35-1.32)$ \\
\hline \multicolumn{3}{|l|}{ Household } \\
\hline \multicolumn{3}{|l|}{ Type of house } \\
\hline Thatched & 1 & 1 \\
\hline Tiled/concrete & $0.75(0.47-1.22)$ & $0.68(0.39-1.18)$ \\
\hline \multicolumn{3}{|l|}{ Income (INR) } \\
\hline$<2,000$ & 1 & 1 \\
\hline $2,000+$ & $1.60(1.09-2.33)$ & $1.59(1.05-2.41)$ \\
\hline
\end{tabular}

*All variables were included in the regression model. $\mathrm{Cl}$ : confidence interval; INR: Indian Rupees. 50 INR was equivalent to 1 US dollar during $2000-2003$.

adjusted hazard ratios of stages II, III and IV cancers compared with stage I cancers of 3.81 (95\% CI: $1.54-9.40), 3.67(95 \%$ CI: $1.56-8.64)$ and $6.74(95 \% \mathrm{CI}: 2.34-19.02)$, respectively during the 12 years of follow-up (Table 2).

Survival after cervical cancer diagnosis was not significantly associated with age at diagnosis, education, occupation, marital status and type of house. Contrary, the 5-year survival estimate of women with higher household income was significantly lower compared to the survival of those with low household income (18.8\% vs. 38.2\%; Table 1$)$ showing an adjusted hazard ratio of 1.59 (95\% CI: $1.05-2.41$; Table 2$)$.

\section{Discussion}

Survival estimates of patients with cervix cancer obtained from population-based data are scarce in low- and middle-income countries because cancer registration is not common and mainly due to problems in follow-up of patients. In a country with limited resources, the survival estimates are obtained from only a fraction of the cancer patients. This problem is aggravated further for rural populations. Here we reported the cervix cancer survival in a rural population covered by a cancer registry and with very few losses to follow-up.

Our observed survival experience of women diagnosed with cervical cancer was poor, with over a half of the women dying within the first 2 years and only about $30 \%$ still alive after 5 years of follow-up. Stage of disease was the strongest determinant of survival. The observed 5-year survival $(32.5 \%)$ in this study is quite similar to that reported by DACR $(35 \%)$ for the cervical cancer cases registered in 2003 from rural Tamil Nadu [14]. The 5-year observed survival of women diag- 


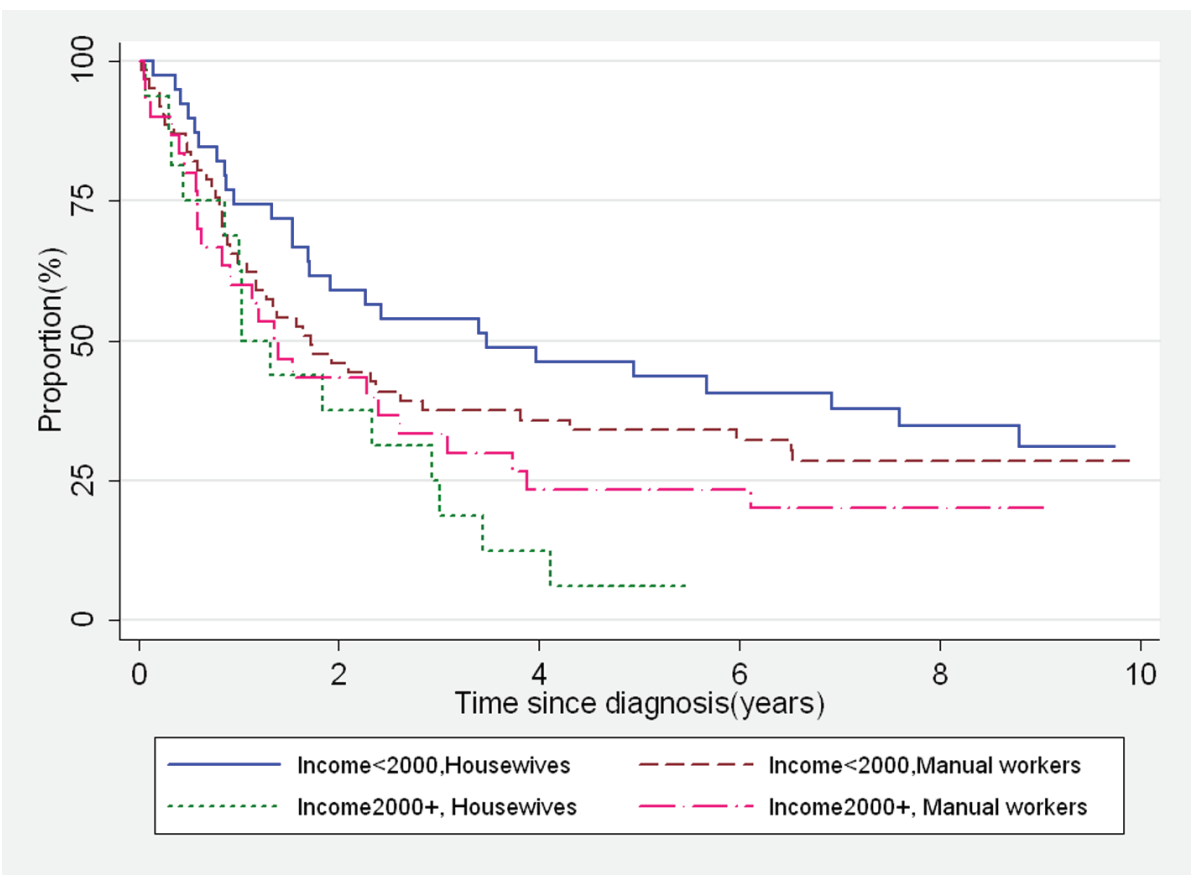

Figure 2. Kaplan-Meier survival curves showing survival of married women with different levels of income and occupation.

nosed with cervical cancer during 1990 - 1999 in the Chennai urban PBCR in Tamil Nadu was 54\%, and 62\% in the Chennai HBCR data of women who received treatment during 2000 2001 [14]. Better cancer health services with more accessibility to diagnosis and treatment result in large variations in survival between different regions of India, particularly in urban vs. rural areas [5]. Delays in treatment due to lack of facilities, support technology, trained personal, financial resources and social or family support affect survival of cervical cancer patients in low-resource settings [6].

Studies from Europe [7, 15] and India [8, 9] showed no association between socio-economic status (SES) and cervical cancer survival, but presence of co-morbid conditions, several clinical and/or pathological factors such as stage were of prognostic importance. These studies also suggest that it may not be possible to clearly establish the role of socio-economic factors in cervical cancer survival within a group of patients who had similar socio-economic characteristics and/or access to treatment and/or inaccessibility to cancer treatment facilities. However, one hospital-based registry study from South India showed that socio-economic factors predict cervical cancer survival along with performance status and clinical stage of disease [1]. Socio-economic factors are in fact not directly linked to survival, but it is directly related to a person's general state of health, nutritional status, attitudes, beliefs and health behavior. It can affect the chances of being early detected, access to or completion of treatment and follow-up and perhaps survival is mediated by all these factors [1].

In addition, the survival analysis by socio-economic factors is problematic by many ways. In our study, we used the baseline information of socio-economic factors. A survival study requires long-term follow-up, and a person's SES can vary over time and it may influence the survival estimates. In this survival analysis, education (formally educated or not) is the most reliable socio-economic indicator because it does not change over time. The available scales for assessing the SES of families in India usually derive from a single piece of information using many components of socio-economic factors [16-18]. The analysis of survival using such single information on SES can only give the variation according to SES in general and that may not be helpful to understand the mechanism of individual components of socio-economic factors which determine the estimates differently. Also, it is possible that the level of individual factors can have different directions of association for different outcomes; for instance education is negatively associated with cervical cancer incidence, but positively associated with breast cancer incidence [19]. Furthermore, the factors which determine the SES of one population may not be applicable to another. Consequently, the assessment of SES should be varied according to the population under study and purpose of the study.

Accordingly, in our study we focused on the variation in survival by different components of SES using several individual and household characteristics. Old age, no schooling, manual occupation, living in thatched houses, and low household income indicated poor SES. When we consider the variable "type of house", we cannot say that the women who lived in tiled/concrete houses had a very high SES; however, we can say that very poor people lived in thatched houses and people whose circumstances were a little better in tiled/concrete houses in rural areas. However, the fact that only $4 \%(1 / 27)$ of women lived in thatched houses were formally educated and had a higher household income, and 78\% (21/27) of women who lived in thatched houses were manual workers further supports the efficiency of this variable to measure poor SES in this population. Today, however, it has become impractical to 
use such a variable to measure SES because thatched houses are gradually disappearing from rural areas through the initiatives of the state and central governments to provide concrete houses for the poor.

Married women and those with an income more than 2,000 INR per month had poor survival. In fact, the difference in survival by income was the only statistical significant result by the SES variables. This seems to be in contradiction with the poor SES hypotheses. As participants provided information on their income group to the interviewer at the time of baseline interview, this opens the possibility of information bias. Only $29 \%(48 / 165)$ of the women reported to have income greater than 2,000 INR and because of this small sample, chance observations (deaths) are also possible.

It was observed from the data that $31.5 \%(46 / 146)$ of married women reported a household income $2,000+$ INR versus $10.5 \%(2 / 19)$ of single women. Conversely $96 \%$ (46/48) of the women reported having household income $2,000+$ INR were currently married. Further, we found that $38 \%(55 / 146)$ of the married women were housewives compared to $26 \%(5 / 19)$ of the single women. The remaining married and single women were manual workers (62\% vs. $74 \%$ ) having their own earnings. It is common in rural India that the married women are not free to spend money without the permission of their husbands, even though they have their own earnings. The housewives reported the income generated by their family members and depend on their husbands/family members to get the money necessary to access healthcare facilities. However, this financial instability often leads to ignorance of health needs. If this is true, the housewives are a poor survival group. To demonstrate this, we further made four categories of married women with different combinations of income and occupation and we could clearly illustrate that those housewives who reported having a higher household income were the poorest survival group (Fig. 2). Hence these findings suggest that a higher household income may not always be a supporting factor for improved survival.

A major limitation of our study was that we did not have information on clinical extent of disease and treatment details of women on a routine basis. Also, the estimates do not take into account deaths due to other causes. However, we have complete and accurate information of all incident cancer cases diagnosed during 2000 - 2006 for the study population, and an adequate and active follow-up of the cancer cases by DACR and mortality registration system and the follow-up of the base population in 2011 - 2012. We had a very minimal number of women with incomplete follow-up status, so we did not have to adjust the estimates for losses. Also we took only those women diagnosed with cancer during 2000 - 2006 to avoid bias in our selection criteria since cervical cancer screening was provided to all 30 - 59 years old women from the control area during 2007 - 2010.

In our study, the variation in hazard with respect to socioeconomic characteristics was not statistically significant probably due to small number of patients. We found that the poor socio-economic characteristics had up to $70 \%$ higher risk of death. For example, there was a $30 \%$ reduction in mortality or a $70 \%$ better survival among those with some schooling compared to no schooling. Even if the difference is not statistically significant, it should not be considered to be zero difference in mortality or survival among the two schooling categories. In addition, the non-significant survival variation by SES may seem less than expected. One explanation may be the general lack of health services in rural India. Specialized service might be too far away or not efficient for cancer diagnosis and treatment.

In conclusion, the cervical cancer survival is likely to be poor also in rural populations of other areas in India and in low- and middle-income countries in general. The only strong determinant was the stage of tumor at diagnosis. Our results further stress the importance of providing prevention and screening opportunities to the women in low-resource settings, and the need to strengthen the quality of healthcare facilities in rural areas.

\section{Acknowledgement}

We acknowledge the Bill \& Melinda Gates foundation for their financial support to our study through the Alliance for Cervical Cancer Prevention.

\section{Conflict of Interest}

None of the authors have any conflicting interest.

\section{Financial Disclosures}

All authors have no financial disclosures to make.

\section{Grant Support}

The original study was financially supported by the Bill \& Melinda Gates foundation.

\section{References}

1. Sankaranarayanan R, Nair MK, Jayaprakash PG, Stanley G, Varghese C, Ramadas V, Padmakumary G, et al. Cervical cancer in Kerala: a hospital registry-based study on survival and prognostic factors. Br J Cancer. 1995;72(4):1039-1042.

2. Dhar M, Lahiri S, Takiar R, Ashok NC, Murthy NS. An indirect study of cancer survival in the context of developing countries. Asian Pac J Cancer Prev. 2008;9(3):479486.

3. Sankaranarayanan R, Swaminathan R. Cancer Survival in Africa, Asia, the Carribbean and Central America. IARC Scientific Publications No. 162. Lyon: International Agency for Research on Cancer; 2011.

4. American Cancer Society. Global Cancer Facts \& Figures. 2nd Edition. Atlanta: American Cancer Society; 2011. Avalabale at: www.cancer.org/research/cancerfactsfig- 
ures/globalcancerfactsfigures/global-facts-figures-2nd-ed (accessed 20 May 2015).

5. Sankaranarayanan R, Swaminathan R, Brenner H, Chen $\mathrm{K}$, Chia KS, Chen JG, Law SC, et al. Cancer survival in Africa, Asia, and Central America: a population-based study. Lancet Oncol. 2010;11(2):165-173.

6. Kaku M, Mathew A, Rajan B. Impact of socio-economic factors in delayed reporting and late-stage presentation among patients with cervix cancer in a major cancer hospital in South India. Asian Pac J Cancer Prev. 2008;9(4):589-594.

7. Coker AL, Du XL, Fang S, Eggleston KS. Socioeconomic status and cervical cancer survival among older women: findings from the SEER-Medicare linked data cohorts. Gynecol Oncol. 2006;102(2):278-284.

8. Nandakumar A, Anantha N, Venugopal TC. Incidence, mortality and survival in cancer of the cervix in Bangalore, India. Br J Cancer. 1995;71(6):1348-1352.

9. Munagala R, Rai SN, Ganesharajah S, Bala N, Gupta RC. Clinicopathological, but not socio-demographic factors affect the prognosis in cervical carcinoma. Oncol Rep. 2010;24(2):511-520.

10. Sankaranarayanan R, Rajkumar R, Arrossi S, Theresa $\mathrm{R}$, Esmy PO, Mahe C, Muwonge R, et al. Determinants of participation of women in a cervical cancer visual screening trial in rural south India. Cancer Detect Prev. 2003;27(6):457-465.

11. Sankaranarayanan R, Esmy PO, Rajkumar R, Muwonge R, Swaminathan R, Shanthakumari S, Fayette JM, et al. Effect of visual screening on cervical cancer incidence and mortality in Tamil Nadu, India: a cluster-randomised trial. Lancet. 2007;370(9585):398-406.
12. Thulaseedharan JV, Malila N, Hakama M, Esmy PO, Cherian M, Swaminathan R, Muwonge R, et al. Effect of screening on the risk estimates of socio demographic factors on cervical cancer - a large cohort study from rural India. Asian Pac J Cancer Prev. 2013;14(1):589-594.

13. Jensen OM, Parkin DM, Maclennan R, Muir CS, Skeet RG. Cancer registration principles and methods. IARC Scientific Publication No. 95. Lyon: IARC Press; 1991.

14. Swaminathan R, Selvakumaran R, Esmy PO, Sampath P, Ferlay J, Jissa V, Shanta V, et al. Cancer pattern and survival in a rural district in South India. Cancer Epidemiol. 2009;33(5):325-331.

15. Schrijvers CT, Mackenbach JP. Cancer patient survival by socioeconomic status in seven countries: a review for six common cancer sites [corrected]. J Epidemiol Community Health. 1994;48(5):441-446.

16. Aggarwal OP, Bhasin SK, Sharma AK, Chhabra P, Aggarwal K, Rajoura OP. A New Instrument (Scale) for Measuring the Socioeconomic Status of Family: Preliminary Study. Indian J Community Med. 2005;30:111-114.

17. Kusum LG. Socio-economic status measurement scale: thirst area with changing concept for socio-economic status. Int Journal of Innovative Research \& Development. 2013;2:139-145.

18. Ramesh Masthi NR, Gangaboraiah, Kulkarni P. An exploratory study on socio economic status scales in a rural and urban setting. J Family Med Prim Care. 2013;2(1):6973.

19. Swaminathan R, Selvakumaran R, Vinodha J, Ferlay J, Sauvaget C, Esmy PO, Shanta V, et al. Education and cancer incidence in a rural population in south India. Cancer Epidemiol. 2009;33(2):89-93. 